\title{
Effect of indium substitution on the electrical and magnetic properties of $\mathrm{Ni}-\mathrm{Zn}$ ferrite
}

\author{
B. B. V. S. Vara Prasad ${ }^{1}$
}

Received: 8 August 2014/Accepted: 5 August 2015/Published online: 21 August 2015

(C) The Author(s) 2015. This article is published with open access at Springerlink.com

\begin{abstract}
Indium-substituted polycrystalline nickel zinc ferrites of different compositions $\mathrm{Ni}_{0.65} \mathrm{Zn}_{0.35} \mathrm{Fe}_{2-x} \mathrm{In}_{x} \mathrm{O}_{4}$ $(x=0.00,0.04,0.08,0.12,0.16,0.2)$ have been prepared by solid-state reaction route. Calcination and sintering of all samples were done in air atmosphere at $950{ }^{\circ} \mathrm{C}$ and $1250{ }^{\circ} \mathrm{C}$, respectively, followed by natural cooling to room temperature. Characterization of the basic composition was done by X-ray diffractometry, saturation magnetization and Curie temperature. The effect of incorporation of larger indium ions in place of iron was studied with the help of saturation magnetization, Curie temperature, permeability, magnetic loss factor, DC electrical resistivity and dielectric properties. The highest magnetization and permeability values of the present system under study are $74.1 \mathrm{emu} / \mathrm{g}$ and 438 for the basic composition (concentration $x=0$ ). The DC electrical resistivity was found to decrease up to $x=0.3$ and increase thereafter. The variation in the electric and magnetic properties of this ferrite system is dominated by the decrease in iron concentration. Characteristic high dielectric constant values were recorded for these bulk ceramic materials.
\end{abstract}

Keywords Ni-Zn ferrite $\cdot$ Magnetization $\cdot$ Curie temperature $\cdot$ DC electrical resistivity $\cdot$ Indium

B. B. V. S. Vara Prasad varaprasadbbvs@gmail.com

1 Department of Physics, MVGR College of Engineering, Chintalavalasa, Vizianagaram 535005, Andhra Pradesh, India

\section{Introduction}

Polycrystalline spinel ferrites are extensively investigated for their usefulness in modern technology. The tunable magnetic and electric properties of ferrites made them better candidates for electronic and electromagnetic applications, like transformer cores, inductors, electromagnetic suppressors (EMI), microwave absorbers, etc [1, 2]. Ferrite crystallizes into face centered cubic spinel, having general formula $\mathrm{AB}_{2} \mathrm{O}_{4}$, where $\mathrm{A}$ and $\mathrm{B}$ are divalent and trivalent metal cations. Ferrite structure comprises two types of sublattices, tetrahedral (A) site and octahedral sites (B). Cations are distributed between these two sublattices in two ways without changing the lattice symmetry. They are, normal (divalent ions occupy A site and trivalent ions occupy B site) and inverse spinel (divalent and trivalent ions distributed between both sites at random). The properties of ferrites strongly depend on not only the method of preparation, but also on the distribution of ions in the crystal lattice.

Several ferrite compositions were studied over the last few decades and some of them are found to be interesting in the application perspective. In the family of spinel ferrites, nickel-zinc mixed ferrites are commercially important magnetic materials because of their high saturation magnetization, high electrical resistivity and low losses. Owing to their moderate permeability values, this mixed ferrite system has some limitations in view point of applications. Thus far, various kinds of substitutions were done on these materials and the effects on their properties are thoroughly studied [3, 4]. Among all those substituted cations, incorporations of several diamagnetic (divalent and trivalent) ions in place of paramagnetic ions into ferrite systems were remarkably improved their magnetic and electric properties [5, 6]. Several researchers investigated trivalent diamagnetic ions 
such as $\mathrm{Al}^{3+}$ and $\mathrm{In}^{3+}$ ions, but mixed results were obtained, which depend on mode of preparation. Furthermore, $\mathrm{In}^{3+}$ ions are substituted at the expense of strong ferromagnetic iron ions which may change the interactions present within the system. Previous research study of indium-substituted nickel-zinc ferrite systems in bulk form showed some interesting variations in the electric and magnetic properties [7-10]. In those systems, reasonable amount of paramagnetic ions were maintained at the expense of strong ferromagnetic ions. The present research study is aimed at investigating the effect of substitution of $\mathrm{In}^{3+}$ ions in place of $\mathrm{Fe}^{3+}$ ions, on magnetic and electric properties. Since the substitution of indium in present system is in the place of strong ferromagnetic $\mathrm{Fe}^{3+}$ ions, which are very crucial in determining the magnetic as well as electric properties of ferrites, the ferrite system may undergo remarkable modifications in its behavior under the influence of electric and magnetic fields.

Because of the intrinsic complexity and less immediate applications, there is less information available on dielectric properties of $\mathrm{Ni}-\mathrm{Zn}$ ferrites. When this ferrite system is incorporated with suitable substituent, it is possible to maintain moderate magnetic and dielectric characteristics simultaneously and these can be potential contenders for microwave absorbers and magneto-dielectric applications. Being diamagnetic, larger in size and variable site occupancy with concentration, prompted the present research study and the variations in the properties have been discussed in the limit of existing understanding.

\section{Experimental details}

Samples having the composition $\mathrm{Ni}_{0.65} \mathrm{Zn}_{0.35} \mathrm{In}_{x} \mathrm{Fe}_{2-x} \mathrm{O}_{4}$ with $x$ values ranging from 0.00 to 0.20 in steps of 0.04 were prepared by solid-state reaction method. The starting materials of analytical reagent grade nickel oxide (99\%), zinc oxide (99\%), indium oxide $(99.5 \%)$ and iron oxide $(99.5 \%)$ in powder form. The solid-state reaction used for the preparation of the samples can be expressed as:

$$
\begin{aligned}
& (0.65) \mathrm{NiO}+(x / 2) \mathrm{In}_{2} \mathrm{O}_{3}+(0.35) \mathrm{ZnO} \\
& +(1-(x / 2)) \mathrm{Fe}_{2} \mathrm{O}_{3} \rightarrow \mathrm{Ni}_{0.65} \mathrm{Zn}_{0.35} \mathrm{In}_{x} \mathrm{Fe}_{2-x} \mathrm{O}_{4}
\end{aligned}
$$

The initial ingredients were weighed in the required proportions using micro-analytical balance. The weighed materials were thoroughly mixed in an agate mortar-pestle for $3 \mathrm{~h}$ in dry medium and $3 \mathrm{~h}$ in acetone medium. This mixture was calcined at $950{ }^{\circ} \mathrm{C}$ for $4 \mathrm{~h}$ in air atmosphere. The pre-sintered powder was again crushed and ground thoroughly for $3 \mathrm{~h}$ in acetone to promote the homogeneity among the mixture. The resulting mixture was made into pellets and toroids under the pressure of 5 and 3 tons/in. ${ }^{2}$, respectively, using $15 \%$ polyvinyl alcohol as binder. The dimensions of the pellets were of $4 \mathrm{~mm}$ thickness and $3 \mathrm{~cm}$ in diameter. The toroids were of $13 \mathrm{~mm}$ inner diameter and $18 \mathrm{~mm}$ outer diameter. Both the pellets and toroids were sintered at $1250{ }^{\circ} \mathrm{C}$ in air atmosphere for $2 \mathrm{~h}$, followed by natural cooling to room temperature. The surfaces of all the samples were polished to remove any oxide layer formed during sintering.

The present series of samples were characterized by Philips X-ray diffractometer with $\mathrm{Cu} k \alpha$ source $(\lambda=1.5418 \AA)$. The interplanar spacing $d_{h k l}$ has been obtained from the intensity versus $2 \theta$ graph of X-ray diffraction pattern using Bragg's law,

$2 d \sin \theta=n \lambda$

The plane indices $(h k l)$ have been assigned to each peak using the X-ray data of the standard spinel structure. Lattice parameter ' $a$ ' has been calculated from each peak using equation, $a=d \sqrt{\left(h^{2}+k^{2+} l^{2}\right)}$ [11]. Nelson-Riley function for the high-intensity peaks calculated using the formula [12]:

$F(\theta)=\frac{1}{2}\left(\frac{\cos ^{2} \theta}{\sin \theta}+\frac{\cos ^{2} \theta}{\theta}\right)$

Accurate determination of lattice parameter has been obtained from the extrapolation of lattice parameter ' $a$ ' versus $F(\theta)$.

Saturation magnetization was measured by Ponderometer (pendulum) method described by Rathenov and Sneok [13]. The ferrite samples used for measurements have the dimensions approximately $2 \mathrm{~mm} \times 3 \mathrm{~mm} \times 3 \mathrm{~mm}$. Curie temperature was measured by the experimental setup described by Soohoo [14].

Inductance $(L)$ measurements on the toroidal samples were carried out using LCR-Q-METER-SORTER at a small voltage of $10 \mathrm{mV}$. The average dimensions of the toroidal samples used in this study are as follows: outer diameter $(\mathrm{OD})=15 \mathrm{~mm}$, inner diameter $(\mathrm{ID})=8 \mathrm{~mm}$; thickness $(h)=4.5 \mathrm{~mm}$. All toroidal samples were wound with 30 SWG enameled copper wire and 30 turns. Initial permeability at $1 \mathrm{kHz}$ frequency and at room temperature was computed using, $\mu_{i}=\frac{L}{L_{0}}$, where $L$ is the inductance of toroidal sample, $L_{0}=4.606 \mathrm{~N}^{2}$ $h \log (\mathrm{OD} / \mathrm{ID}) 10^{-9}$ Henry is the air core inductance, and $N$ is the number of turns.

The DC resistivity of the ferrite samples is measured with two-probe method. In this method, the sample is placed in between two spring-loaded copper electrodes connected to Digital Nano-ammeter model DNM-121. The freshly ground flat surfaces of the samples were coated with conducting (air-drying) silver paint and placed in an oven at $100{ }^{\circ} \mathrm{C}$ for $30 \mathrm{~min}$ to ensure good ohmic contacts. The electric current in the samples was measured with the 
help of digital nano-ammeter by applying a constant field of $1 \mathrm{~V} / \mathrm{cm}$ between the parallel surfaces of the sample.

The present series of samples were investigated for dielectric properties by using LCR-Q-METER-SORTER (APLAB model 4912) at $1 \mathrm{kHz}$ frequency and at room temperature. If $C$ is the capacitance of the sample, $d$ its thickness and $A$ the surface area, then, dielectric constant $\varepsilon^{\prime}=C d / \varepsilon_{0} A$, where $\varepsilon_{0}$ is the dielectric constant of the air. The dielectric loss factor $\varepsilon^{\prime \prime}$ has been calculated from the relation: $\varepsilon^{\prime \prime}=\varepsilon^{\prime} \tan \delta$, where $\tan \delta$ is the loss tangent.

\section{Results and discussion}

\section{Characterization}

X-ray diffraction patterns of all the indium-substituted nickel-zinc ferrite samples have the standard planes (220), (311), (222), (400), (422), (511), (440) and (533), as well as no detectable additional peaks, which confirm the formation of single phase cubic spinel structure. X-ray diffraction pattern of the basic sample $\mathrm{Ni}_{0.65} \mathrm{Zn}_{0.35} \mathrm{Fe}_{2} \mathrm{O}_{4}$ is shown in Fig. 1a.

The characteristic $d$-spacings of the basic sample are in good agreement with values reported in standard JCPDS cards. The lattice constant for the basic sample was determined using Nelson-Riley extrapolation method. Nelson-Riley plot for the basic sample is shown in Fig. 1b.

The experimentally observed lattice constant (8.3738 ̊) of the basic sample is in agreement with the reported value (8.382 $\AA$ ) of the same composition [15].

\section{Saturation magnetization}

Magnetization of ferrites is strongly dependent on the exchange interactions between cations present at $\mathrm{A}$ and $\mathrm{B}$ sites. These exchange interactions are A-B, B-B and A-A interactions, either positive or negative. The parallel arrangement of cations is represented by positive and negative sign denotes anti-parallel arrangement of cations. These interactions depend on magnitude, sign, the extent and form of overlap of the $3 d$ electron wave functions of the neighboring ions. The strength of the interaction is proportional to the distance between ions and the cations present in the ferrite system. Therefore, when the system is altered with larger/smaller cations it may influence the magnetization. Magnetization of ferrites also can be explained on Neel's theory of ferrimagnetism (two sub lattice model) [16]. According to this model, the magnetization of ferrites depends on the magnetic moments of two sublattices [tetrahedral (A) and octahedral (B) sites]. From Neel's two sublattice model, the expression for the total net magnetization of the ferrite structure $M=\left|M_{\mathrm{B}}-M_{\mathrm{A}}\right|$, where $M_{\mathrm{B}}$ and $M_{\mathrm{A}}$ are magnetizations of A and B sub lattices, respectively. The variation of saturation magnetization and Curie temperature with the increase of indium concentration is shown in Fig. 2.

Saturation magnetization of the present series of samples is observed to be decreased from $x=0$ to 0.2 with increase in indium concentration, but the decrease is not perfectly linear. From the available literature [10], indium has variable site occupancy. For lower concentrations of indium, $\mathrm{In}^{3+}$ ions migrate to tetrahedral sites leading to the dilution of A-sublattice and decrease in the magnetic moment of the A-sublattice. This will result in the modifications of the exchange interactions. Moreover, the substitution of diamagnetic $\mathrm{In}^{3+}$ ions at the expense of ferromagnetic $\mathrm{Fe}^{3+}$ ions reduces number of magnetic ions to non-magnetic ions ratio at octahedral sites, results in the dilution in the net magnetic moment of the ferrite system as a whole, that is, there is a decrease of $0.04 \times 5=0.2 \mathrm{Bohr}$ magnetons per one formula unit. At higher concentrations of $\mathrm{In}^{3+}$ ions, because of the small octahedral preference of indium, these ions move to octahedral sites leading to the migration of $\mathrm{Fe}^{3+}$ ions from octahedral site to tetrahedral site, reducing octahedral magnetic moment $\left(M_{\mathrm{B}}\right)$. Thus, the net magnetic moment of the system decreases. Furthermore, the incorporation of larger indium ions (0.62 and
Fig. 1 X-ray diffraction pattern and Nelson-Riley graph of $\mathrm{Ni}_{0.65} \mathrm{Zn}_{0.35} \mathrm{Fe}_{2} \mathrm{O}_{4}$
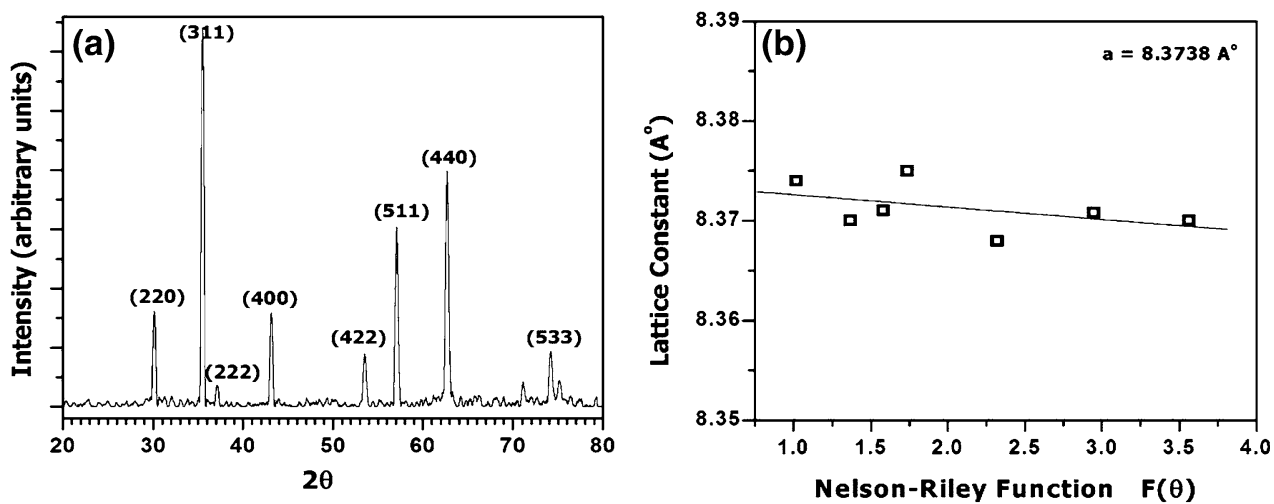


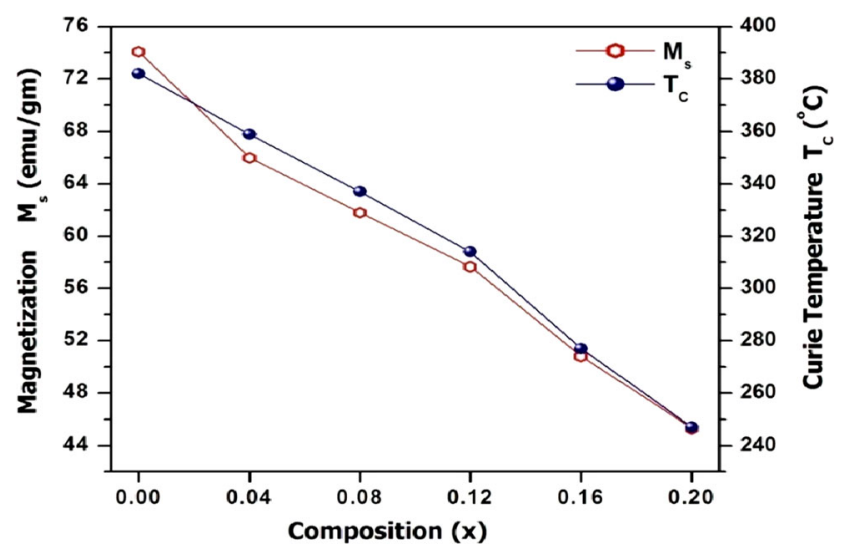

Fig. 2 Variation of saturation magnetization and Curie temperature with indium concentration for $\mathrm{Ni}_{0.65} \mathrm{Zn}_{0.35} \mathrm{Fe}_{2-x} \mathrm{In}_{x} \mathrm{O}_{4}$

$0.8 \AA$ in $\mathrm{A}$ and $\mathrm{B}$ sites) in place of iron ions (0.49 and $0.645 \AA$ in $A$ and $B$ sites), makes the sublattices to stretch, resulted in the increase in distances between magnetic ions of $A$ and $B$ sites. As a result, the interaction between $A$ and $\mathrm{B}$ site ions (A-B exchange interactions) weakens, which is evident by the fall of Curie temperature with increasing indium content. The saturation magnetization $(74.1 \mathrm{emu} / \mathrm{g})$ and Curie temperature $\left(382{ }^{\circ} \mathrm{C}\right)$ of the basic sample are well in agreement with the reported values [17].

\section{Permeability and magnetic loss}

The variation of permeability and magnetic loss factor with composition at a standard frequency $1 \mathrm{kHz}$ are shown in Fig. 3. The initial permeability slowly decreases from basic composition $x=0$ to $x=0.04$, after there is a sharp fall in the vicinity of $x=0.08$, followed by a gradual decrease up to the last sample. The decrease in permeability can be explained as follows.

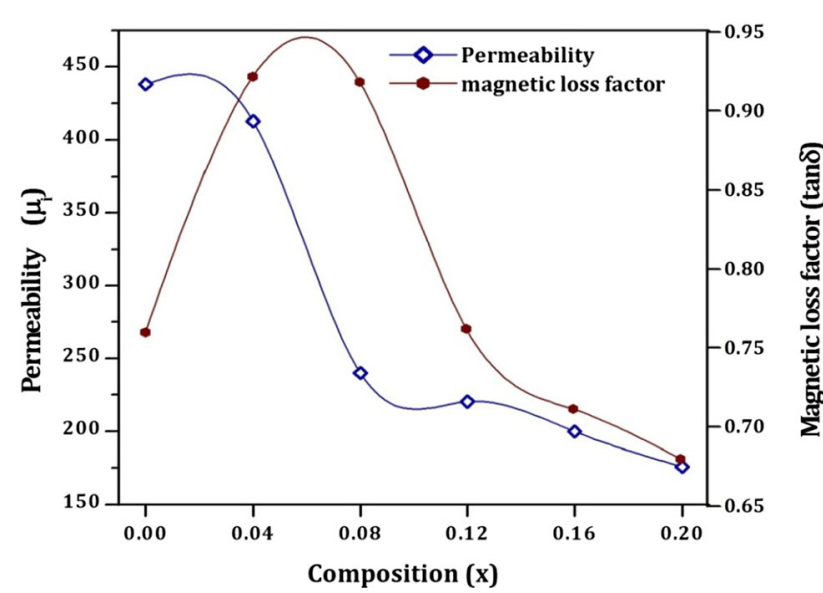

Fig. 3 Variation of initial permeability and magnetic loss factor with indium concentration for $\mathrm{Ni}_{0.65} \mathrm{Zn}_{0.35} \mathrm{Fe}_{2-x} \mathrm{In}_{x} \mathrm{O}_{4}$
In general, $\mathrm{Fe}^{3+}$ ions occupy both tetrahedral and octahedral sites. From the available literature, it is known that the anisotropy contribution of the $\mathrm{Fe}^{3+}$ is positive for tetrahedral site and negative for octahedral site [18]. Since, the $\mathrm{Fe}^{3+}$ ions distributed over the two lattices, the net anisotropy is taken from the relative contribution of these ions towards anisotropy. For lower concentration of indium $(x=0.04)$, they prefer to occupy tetrahedral sites [19], thus, $\mathrm{Fe}^{3+}$ ions move to octahedral sites, consequently, decrease in the net anisotropy. Therefore, an increase in permeability is expected, but the decrease in magnetization $(8.1 \mathrm{emu} / \mathrm{g})$ dominates and resulted in the slight decrease of permeability (438-412.9). After $x=0.04$, huge drop in permeability was observed and it is attributed to displacement of indium ions towards octahedral sites. With the increase in diamagnetic indium substitution, $\mathrm{In}^{3+}$ ions occupy octahedral sites, thus decrease in the concentration of $\mathrm{Fe}^{3+}$ ions at $\mathrm{B}$ site compared to A site. As a result, the net magnetic anisotropy contribution from iron ions distributed over two sites increases, so as the magnetic crystalline anisotropy constant $K_{1}$. According to the relation $\mu_{i} \alpha\left(M_{\mathrm{s}}^{2} D_{\mathrm{m}}\right) / K_{1}$ [6], permeability is inversely proportional to magneto crystalline anisotropy constant. So, the increase in anisotropy and decrease in magnetization $M_{\mathrm{s}}$ is responsible for the decrease in permeability. In this present system, low concentration of $\mathrm{Fe}^{2+}$ ions are present, which are evident from the observed fall in resistivity values and the contribution of $\mathrm{Fe}^{2+}$ ions to the anisotropy is positive. But it is not taken into consideration, because the amount of $\mathrm{Fe}^{2+}$ ions present in $\mathrm{B}$ site is less than amount of $\mathrm{Fe}^{3+}$ ions moved to the tetrahedral site. The variation in magnetic loss factor is attributed to the formation of $\mathrm{Fe}^{2+}$ ions in octahedral sites, which are more at $x=0.04$ and 0.08 . This is supported by the decrease in resistivity.

\section{DC electrical resistivity}

The variation of dc electrical resistivity with composition is shown in Fig. 4.

The resistivity of the substituted indium $(8.37 \mu \Omega \mathrm{cm})$ is less than the replaced iron $(9.61 \mu \Omega \mathrm{cm})$ [20]; thus, a decrease in resistivity of the whole series is expected. But the resistivity is decreased initially up to $x=0.08$ and then increased up to last composition. The variation in resistivity can be explained by the following manner. In general, the resistivity can be explained on the basis of electron hopping mechanism. The transfer of electron between same/different elements having different states is called hopping. It is assumed that $\mathrm{Fe}^{3+}$ can occupy both $\mathrm{A}$ and $\mathrm{B}$ sites, but it should be noted that $\mathrm{Fe}^{2+}$ ions at octahedral sites are responsible for electrical conduction [21]. 


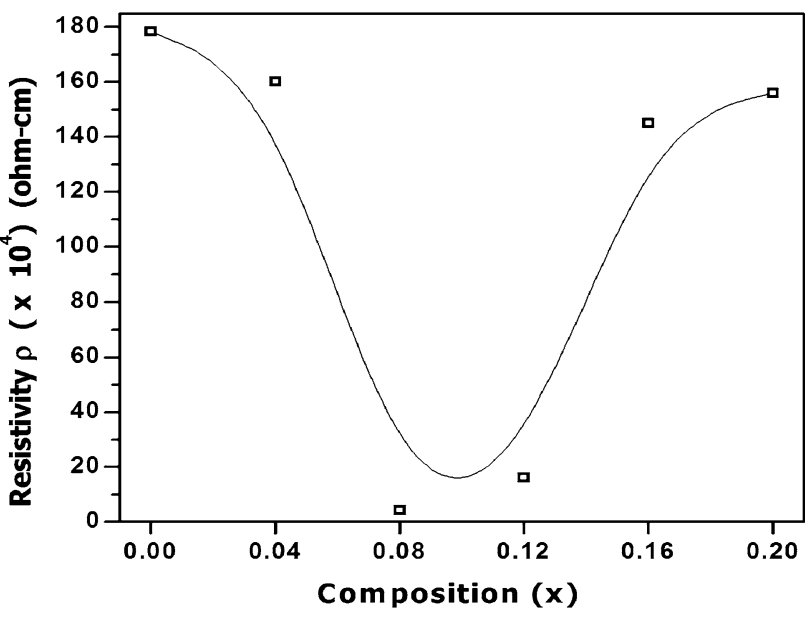

Fig. 4 Variation of DC electrical resistivity with indium concentration for $\mathrm{Ni}_{0.65} \mathrm{Zn}_{0.35} \mathrm{Fe}_{2-x} \mathrm{In}_{x} \mathrm{O}_{4}$

At lower concentrations indium ions will occupy A site [10], thus there is a movement of $\mathrm{Fe}^{3+}$ ions from $\mathrm{A}$ to $\mathrm{B}$ site, thus increasing concentration of $\mathrm{Fe}^{3+}$ ions at $\mathrm{B}$ site. At high sintering temperatures, zinc volatilization occurs and they escape from the samples in the form of $\mathrm{ZnO}$, thus, creating oxygen vacancies during the process [22]. In this situation, to maintain charge balance, formation of $\mathrm{Fe}^{2+}$ ions takes place, resulting in increasing the hopping possibility and this may be responsible for the decrease in resistivity up to $x=0.08$. At higher concentrations most of the indium ions occupy $\mathrm{B}$ site leading to a less concentration of $\mathrm{Fe}^{3+}$ at $\mathrm{B}$ site, that is, less possibility of having $\mathrm{Fe}^{2+}$ ions. Thus, $\mathrm{In}^{3+}$ ions in the $\mathrm{B}$ site hinder the electron exchange between ions $\mathrm{Fe}^{2+}$ and $\mathrm{Fe}^{3+}$ located in equivalent crystallographic positions in the lattice and contribute to the increase in the resistivity. As a whole, the resistivity of the entire series of substituted samples was found less than the resistivity of basic composition $178.4 \times 10^{4} \Omega \mathrm{cm}$. The resistivity values of the present series of samples are greater than two orders of the reported indium-substituted $\mathrm{Ni}-\mathrm{Zn}$ ferrite with small concentration of titanium [10]. The increase in the resistivity of the present system may be due to the absence of titanium.

\section{Dielectric properties}

Ferrites, which are heterogeneous dielectric ceramic materials, mainly because of the formation of $\mathrm{Fe}^{2+}$ ions during sintering process, giving rise to dielectric polarization producing large dielectric constants. Especially in $\mathrm{Ni}-$ $\mathrm{Zn}$ mixed ferrite system, high dielectric constants are reported [23, 24], because of the formation of $\mathrm{Fe}^{2+}$ ions at the expense of zinc volatilization. Another cause is the presence of higher conductivity phases (grains) in the insulating matrix (grain boundaries) producing a local

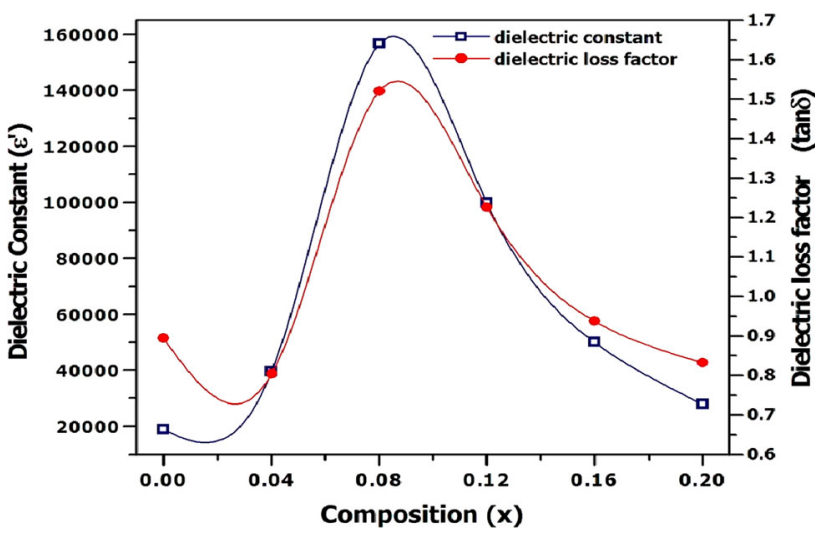

Fig. 5 Variation of dielectric constant and loss factor with indium concentration for $\mathrm{Ni}_{0.65} \mathrm{Zn}_{0.35} \mathrm{Fe}_{2-x} \mathrm{In}_{x} \mathrm{O}_{4}$

accumulation of charge under the influence of an applied electric field. The interface between the grains could be grain boundaries, intra-granular pores or voids which act as scattering centers for electrons. The dielectric behavior in ferrites could be explained on the basis hopping conduction mechanism which is similar to that of mechanism of dielectric polarization. Many researchers have established a strong correlation between conduction mechanism and dielectric constant of ferrites [25]. In these studies they have explained the dielectric behavior on the basis of number of available $\mathrm{Fe}^{2+}$ ions in the octahedral sites. The electron exchange $\mathrm{Fe}^{2+} \leftrightarrow \mathrm{Fe}^{3+}$ results in a local displacement of electrons in the direction of applied electric field which determines the extent of polarization so as the dielectric constants of ferrites.

The variation of dielectric constant and loss factor at a frequency $1 \mathrm{kHz}$ is shown in Fig. 5.

The observed variation in dielectric constant with indium concentration could be explained by local displacement of charge carriers in presence of applied electric field and variation of resistivity. At lower indium concentration, due to oxygen ion vacancies in the samples, there is an availability of additional electron and electron exchange between $\mathrm{Fe}^{2+} \leftrightarrow \mathrm{Fe}^{3+}$. Thus, the excess charge carriers experience drift from the applied of field and leading to dielectric polarization. Which is clearly displayed in the dielectric constant values, especially in the vicinity of $x=0.08$. At this concentration, lowest resistivity (highest conductivity) of the entire series was observed. At higher indium concentration, iron ions are less available for hopping, thus the electron is obstructed by larger indium ions in B site and the electrons/ions cannot follow the field resulting in a decrease of dielectric constant. This is well supported by the increase in resistivity. The high dielectric constant values are characteristic for bulk ferrites. These kinds of values were earlier reported by the author [23] for the same system with cadmium substitution and comparable results were also showed in similar system in bulk form 
[24]. Similar behavior is observed in dielectric loss factor also. Dielectric loss is attributed to several factors such as microstructure, defects, stoichiometry, $\mathrm{Fe}^{2+}$ concentration, etc. The variable $\mathrm{Fe}^{2+}$ concentration is responsible for dielectric loss in the present series of samples.

\section{Conclusion}

Indium-substituted $\mathrm{Ni}-\mathrm{Zn}$ ferrite system was prepared by conventional double sintering solid-state reaction route. The basic composition was characterized with X-ray diffraction pattern and the observed characteristic peaks confirmed the method adopted for the process of ferrites was the correct one. The saturation magnetization and Curie temperature have been decreased with indium content. Firing the samples at elevated temperatures resulted in the formation of $\mathrm{Fe}^{2+}$ ions, affecting electrical properties. DC electrical resistivity was decreased initially and then increased up to the last composition. Dielectric constant and loss factor increased up to $x=0.08$ then decreased afterwards. Highest dielectric constant is observed at $x=0.08$ due to the presence of more $\mathrm{Fe}^{2+}$ ions. The dielectric constant and loss factor followed a trend opposite to the resistivity. The noticeable increase in permeability, dielectric constant and moderate resistivity of the samples $x=0.08$ and 0.12 were observed and displayed the typical characteristics of magneto-dielectric and microwave-absorbing materials.

Open Access This article is distributed under the terms of the Creative Commons Attribution 4.0 International License (http://crea tivecommons.org/licenses/by/4.0/), which permits unrestricted use, distribution, and reproduction in any medium, provided you give appropriate credit to the original author(s) and the source, provide a link to the Creative Commons license, and indicate if changes were made.

\section{References}

1. Horvath, M.P.: Microwave applications of soft ferrites. J. Magn. Magn. Mater. 215-216, 171-183 (2000)

2. Zhao, D.-L., Lv, Q., Shen, Z.-M.: Fabrication and microwave absorbing properties of $\mathrm{Ni}-\mathrm{Zn}$ spinel ferrites. J. Alloy. Compd. 480, 634-638 (2009)

3. Rezlescu, E., Sachelarie, L., Popa, P.D., Rezlescu, N.: Effect of substitution of divalent ions on the electrical and magnetic properties of $\mathrm{Ni}-\mathrm{Zn}-\mathrm{Me}$ ferrites. IEEE Trans. Magn. 36(6), 3962-3967 (2000)

4. Singh, Amarendra K., Goel, T.C., Mendiratta, R.G., Thakur, O.P., Prakash, C.: Magnetic properties of Mn-substituted Ni-Zn ferrites. J. Appl. Phys. 92, 3872 (2002)
5. Caltun, O.F., Spinu, L., Stancu, A.: Magnetic properties of high frequency $\mathrm{Ni}-\mathrm{Zn}$ ferrites doped with $\mathrm{CuO}$. IEEE Trans. Magn. 37(4), 2353-2355 (2001)

6. Prasad, M.S.R., Prasad, B.B.V.S.V., Rajesh, B., Rao, K.H., Ramesh, K.V.: Magnetic properties and DC electrical resistivity studies on cadmium substituted nickel-zinc ferrite system. J. Magn. Magn. Mater. 323, 2115-2121 (2011)

7. Sankpal, A.M., Suryavanshi, S.S., Kakatkar, S.V., Tengshe, G.G., Patil, R.S., Chaudhari, N.D., Sawant, S.R.: Magnetization studies on aluminium and chromium substituted Ni-Zn ferrites. J. Magn. Magn. Mater. 186, 349-356 (1998)

8. Kakatkar, S.V., Kakatkar, S.S., Patil, R.S., Maskar, P.K., Sankapal, A.M., Suryawanshi, S.S., Chaudhari, N.D., Sawant, S.R.: X-ray and bulk magnetic properties of aluminium substituted ferrites. J. Magn. Magn. Mater. 159, 361-366 (1996)

9. Sankpal, A.M., Kakatkar, S.V., Chaudhari, N.D., Patil, R.S., Sawant, S.R., Suryavanshi, S.S.: Initial permeability studies on $\mathrm{Al}^{3+}$ and $\mathrm{Cr}^{3+}$ substituted Ni Zn ferrites. J. Mater. Sci. Mater. Electron. 9, 173-179 (1998)

10. Parvatheeswara Rao, B., Rao, K.H.: Distribution of $\operatorname{In}^{3+}$ ions in indium-substituted Ni-Zn-Ti ferrites. J. Magn. Magn. Mater. 292, 44-48 (2005)

11. Cullity, B.D.: Elements of X-ray diffraction, 2nd edn. AdissonWesley, USA (1978)

12. Nelson, J.B., Riley, D.P.: An experimental investigation of extrapolation methods in the derivation of accurate unit-cell dimensions of crystals. Proc. Phys. Soc. 57, 160 (1945)

13. Rathenau, G.W., Snoek, J.L.: Apparatus for measuring magnetic moments. Philip Res. Rep. 1, 239 (1946)

14. Soohoo, R.F.: Theory and Application of Ferrites, vol. 6. Prentice Hall, Englewood Cliffs (1960)

15. Leung, L.K., Evans, B.J., Morrish, A.H.: Low-temperature Mössbauer study of a nickel-zinc ferrite: $\mathrm{Zn}_{x} \mathrm{Ni}_{1-x} \mathrm{Fe}_{2} \mathrm{O}_{4}$. Phys. Rev. B 8, 29 (1973)

16. Neel, L.: Magnetic properties of ferrites: ferrimagnetism and antiferromagnetism. Ann. Phys. (Paris) 3, 137 (1948)

17. Dionne, G.F., West, R.G.: Magnetic and dielectric properties of the spinel ferrite system $\mathrm{Ni}_{0.65} \mathrm{Zn}_{0.35} \mathrm{Fe}_{2-x} \mathrm{Mn}_{x} \mathrm{O}_{4}$. J. Appl. Phys. 61, 3868 (1987)

18. Singh, M., Sud, S.P.: Controlling the properties of magnesiummanganese ferrites. Mater. Sci. Eng. B 83(1-3), 180-184 (2001)

19. Parvatheeswara Rao, B., Rao, K.H.: Effect of sintering conditions on resistivity and dielectric properties of $\mathrm{Ni}-\mathrm{Zn}$ ferrites. J. Mater. Sci. 32(22), 6049-6054 (1997)

20. Kittel, C.: Introduction to Solid State Physics, 5th edn. Wiley, New York (1976)

21. Smit, J., Wijn, H.P.J.: Les Ferrites. Dunod, Paris (1961)

22. Prinya Sainamthip, V.R.W.: Amarakoon role of zinc volatilization on the microstructure development of manganese zinc ferrites. J. Am. Ceram. Soc. 71(8), 644-648 (1988)

23. Koops, C.G.: On the dispersion of resistivity and dielectric constant of some semiconductors at audio frequencies. Phys. Rev. 83, $121(1951)$

24. Vara Prasad, B.B.V.S.: Cation distribution, structural and electric studies on cadmium substituted nickel-zinc ferrite. Mod. Phys. Lett. B 28(19), 1450155 (13 pages) (2014)

25. Khalid Mujasam Batoo: Study of dielectric and impedance properties of Mn ferrites. Phys. B 406, 382-387 (2011) 